Preprint typeset in JHEP style - PAPER VERSION

CERN-PH-TH/2006-232

FNT/T-2006/09

hep-ph/0611129

November 2006

\title{
Matching matrix elements and shower evolution for top-quark production in hadronic collisions
}

\author{
Michelangelo L. MANGANO \\ CERN, TH-PH, CH 1211 Geneva 23, Switzerland \\ E-mail: michelangelo.mangano@cern.ch \\ Mauro MORETTI \\ Dipartimento di Fisica, Università di Ferrara, and INFN, Ferrara, Italy \\ E-mail: mauro.moretti@fe.infn.it

\section{Fulvio PICCININI} \\ INFN, Sezione di Pavia, v. A. Bassi 6, I 27100, Pavia, Italy \\ E-mail: fulvio.piccinini@pv.infn.it

\section{Michele TRECCANI} \\ Dipartimento di Fisica, Università di Ferrara, and INFN, Ferrara, Italy \\ E-mail: michele.treccani@fe.infn.it
}

\begin{abstract}
We study the matching of multijet matrix elements and shower evolution in the case of top production in hadronic collisions at the Tevatron and at the LHC. We present the results of the matching algorithm implemented in the ALPGEN Monte Carlo generator, and compare them with results obtained at the parton level, and with the predictions of the MC@NLO approach. We highlight the consistency of the matching algorithm when applied to these final states, and the excellent agreement obtained with MC@NLO for most inclusive quantities. We nevertheless identify also a remarkable difference in the rapidity spectrum of the leading jet accompanying the top quark pair, and comment on the likely origin of this discrepancy.
\end{abstract}

Keywords: Hadronic Collisions, Jets, top quark production, Monte Carlo. 


\section{Contents}

1. Introduction 1

2. Review of the matching algorithm 2

3. Consistency studies of the matching algorithm 3

3.1 Comparison with parton-level results 4

$\begin{array}{lll}3.2 & \text { Impact of higher-order parton processes } & 7\end{array}$

$\begin{array}{lll}3.3 & \text { Study of the generation and matching systematics } & 10\end{array}$

4. Comparisons with MC@NLO 14

4.1 Spin correlations in top decays 20

5. Conclusions 21

\section{Introduction}

Following the excellent performance and large statistics accumulated by the Tevatron collider, and in anticipation of the forthcoming beginning of the LHC operations, the recent years have witnessed an impressive progress in the development of improved tools for the simulation of the complex final states produced in hard hadronic collisions [1,2]. The leading themes of these advances have been the inclusion of next-to-leading-order (NLO) matrix elements in the shower Monte Carlo (MC) codes $[3,4]$, and the consistent merging of shower MCs with the leading-order (LO) calculations for final states with many hard partons [5]-[13]. These two directions provide alternative approaches to the common goal of improving the accuracy of the description of multijet final states. In the first case, known as MC@NLO, the emphasis is on achieving the NLO accuracy in the description of the inclusive rates for a given final state $F$, accompanied by the exact LO description of the emission of one extra jet $(F+$ jet $)$. Concrete implementations so far include the cases where $F$ is a pair of gauge bosons [3, 14], a heavy-quark pair [15, 17], a single vector boson or a Higgs [16], and single top [18]. In the second approach, the goal is to maintain a consistent leading-logarithmic (LL) accuracy in the prediction of a final state $F$ accompanied by a varying number of extra jets. This means that the cross sections for each jet multiplicity $N$ are calculated using the LO matrix elements for $N$ hard partons, followed by the full shower evolution obtained with a shower MC. The removal of double counting of jet configurations that would appear both from hard emissions during the shower evolution and from the inclusion of the higher-order matrix elements is achieved in the MC@NLO technique through the explicit subtraction from the NLO matrix elements of the $\mathcal{O}\left(\alpha_{s}\right)$ emission probabilities generated by the shower. It is instead achieved in the second approach via the inclusion in the LO matrix elements of the appropriate Sudakov form factors, and by vetoing shower evolutions leading to multiparton final states already described by the matrix element 
computation. This procedure is known as a matching algorithm for matrix elements and parton shower. While the LL matching algorithm approach cannot be expected to accurately reproduce the inclusive NLO cross section and its stability w.r.t. scale variations, the exact LO description of higher multiplicity partonic final states will give it a better accuracy for the distributions of two or more jets produced in addition to $F$. In this respect the two approaches are complementary, both in goals and in expected performance. Furthermore, we expect that they should give comparable results for $F$ and $F+1$ jet inclusive distributions, up to a possible overall NLO $K$ factor to be applied to the LL results.

The aim of this note is to compare the results of the two approaches in the case of top quark production. We use the code developed by Frixione et al [15] to generate the MC@NLO results, and the ALPGEN code [19] with the so-called MLM matching [9, 10, 13] to generate the LL distributions. Section 2 will briefly review this algorithm. Section 3 will show in detail the results of the ALPGEN calculations for $t \bar{t}$ production, presenting a series of consistency checks of the apporach and discussing its systematic uncertainties. Section 4 covers the comparison between ALPGEN and MC@NLO, and Section 5 will present our conclusions.

\section{Review of the matching algorithm}

The main requirements and features of a good matching algorithm are:

- The removal of double counting of equivalent phase-space configurations.

- The ability to merge together samples with different hard-parton multiplicity, generating fully inclusive event samples leading to predictions for physical observables that are independent of the partonic generation cuts. In particular, this means that the physical definition of a jet will not be a required input for the generation, and that any definition of jet can be used at the analysis level.

The algorithm used in this work is defined by the following rules (for more details, see [10]):

1. Generate parton-level configurations for all final-state parton multiplicities up to $N$, with partons constrained by

$$
p_{T}^{\text {part }}>p_{T}^{\text {min }}, \quad\left|\eta_{\text {part }}\right|<\eta_{\text {max }}, \quad \Delta R>R_{\text {min }},
$$

where $p_{T}^{\text {part }}$ and $\eta_{\text {part }}$ are the transverse momentum and pseudorapidity of the light final-state partons, and $\Delta R$ is their minimal separation in the $(\eta, \phi)$ plane. Each of the samples will be called the $n$-parton sample $(n=0, \ldots, N)$, and the one with $n=0$ will also be referred to as the lowest-order sample.

2. Perform the shower evolution on each $n$-parton sample, using e.g. the standard HERWIG [20] or PYTHIA [21] shower MC codes.

3. For each event, apply a cone jet algorithm to all partons resulting from the shower evolution, before hadronization. We call clusters the resulting jets; they are defined by a minimum $E_{T}$,

$E_{T}^{\text {clus }}$, and by a jet cone size $R_{\text {clus }}$, parameters which are related but not necessarily identical to the partonic generation parameters $p_{T}^{\min }$ and $R_{\text {min }}$.

4. Associate each parton from the PL event to one and only one of the reconstructed clusters: 
- Starting from the highest- $p_{T}$ parton, select the cluster with minimum distance $\Delta R$ from it; if $\Delta R<R_{\text {match }}$, where $R_{\text {match }}$ is a fixed parameter called the matching radius, then we say that the parton is matched.

- Remove the cluster from the list of clusters, go to the next parton and iterate until all hard partons have been processed. Since clusters are removed from the list after they are matched, a given cluster can only be matched to a single parton.

5. If each parton is matched to a cluster, the event "matches", and is kept for further scrutiny, else it is rejected.

6. In the case of $n<N$, matched events with a number of clusters $N_{\text {clus }}>n$ are rejected. This leaves a exclusive sample with $N_{\text {clus }}=n$.

7. If $n=N$, the largest parton multiplicity for which we generated PL events, accept matched events where $N_{\text {clus }}>N$, provided the non-matched clusters (namely those remaining in the cluster list after all clusters matching partons have been removed) are softer than each of the matched clusters.

8. After matching, combine the exclusive event samples from each partonic multiplicity $n=$ $0, \ldots, N-1$ and the inclusive event sample with $n=N$ into a single event sample, which will define the fully inclusive sample.

We shall use the implementation of this matching algorithm given in the ALPGEN code, but the algorithm can be implemented in any other matrix-element based programme. For the shower evolution we use HERWIG [20], version 6.510 [22]. For a more direct comparison with the PL results, we stopped the evolution after the perturbative phase, and our results do not therefore include the effects of cluster splitting and hadronization.

\section{Consistency studies of the matching algorithm}

In this section we study the overall consistency of the matching algorithm applied to $t \bar{t}$ final states. We need to verify the following:

- Inclusive distributions obtained after the matching should reproduce inclusive quantities as calculated at the PL.

- Physical observables should be stable w.r.t. variations of the phase-space cuts applied in the generation of the PL samples (the generation parameters), and w.r.t. variations of the parameters chosen for the parton-jet matching and extra-jet vetoes (the matching parameters).

To start with, we introduce our sets of generation and matching parameters. We shall consider $t \bar{t}$ production at the Tevatron $(p \bar{p}$ collisions at $\sqrt{S}=1.96 \mathrm{TeV})$ and at the LHC ( $p p$ collisions at $\sqrt{S}=14 \mathrm{TeV}$ ). The generation parameters for the light partons are defined by the kinematical cuts given in eq.(2.1), while no cuts are applied to the top quarks. The numerical values chosen for the generation of the default event samples at the Tevatron (LHC) are given by: $p_{T}^{m i n}=20(30) \mathrm{GeV}$,

$\eta_{\max }=4(5)$ and $R_{\min }=0.7(0.7)$. The top quarks are assumed to be stable (with the exception of the study of spin correlations in Section 4.1), and therefore all jets coming from the decay of top quarks are neglected. 


\begin{tabular}{|l|ll|lll|}
\hline Collider & LO & NLO & $0_{e x c}$ & $1_{\text {inc }}$ & $0_{\text {exc }}+1_{\text {inc }}$ \\
\hline Tevatron & $\mathbf{4 . 3 7}$ & 6.36 & 3.42 & 0.78 & $\mathbf{4 . 2 0}$ \\
LHC & $\mathbf{4 7 1}$ & 769 & 217 & 252 & $\mathbf{4 6 9}$ \\
\hline
\end{tabular}

Table 1: Cross sections (in pb) at the LO $\left(\mathcal{O}\left(\alpha_{s}^{2}\right)\right)$ and NLO $\left(\mathcal{O}\left(\alpha_{s}^{3}\right)\right)$ PL, compared with the rates of the $S_{1}$ samples.

For all generations we chose the parton distribution function set MRST2001J[23], with renormalization and factorization scales squared set equal to the sum of the squared transverse masses of the final state partons: $\mu_{\mathrm{R}}^{2}=\mu_{\mathrm{F}}^{2}=\sum_{i=t, \bar{t}, \mathrm{jets}}\left[m_{i}^{2}+\left(p_{T}^{i}\right)^{2}\right]$.

The default matching parameters are defined by the following set of relations:

$$
E_{T}^{\text {clus }}=\max \left(p_{T}^{\text {min }}+5 \mathrm{GeV}, 1.2 \times p_{T}^{\text {min }}\right), \quad \eta_{\text {max }}^{\text {clus }}=\eta_{\text {max }}, \quad R_{\text {match }}=1.5 \times R_{\text {min }},
$$

where $E_{T}^{\text {clus }}$ is the minimum transverse energy of the jet clusters used for the jet-parton matching, $\eta_{\text {max }}^{\text {clus }}$ is their maximum $|\eta|$ and $R_{\text {match }}$ is the maximum separation between parton and jet cluster required for the parton-jet pair to match. Jet clusters are defined by the cone algorithm provided by the GETJET package [24], which represents a simplified jet cone algorithm a la UA1. Variations of these default choices will be defined when exploring the parameter dependence of the results.

The event samples emerging after the showering, matching and veto are defined by the multiplicity of the light partons present in the PL sample, $n$, and by the presence (or absence) of the extra-jet veto: $n_{\text {exc }}\left(n_{\text {inc }}\right)$. For example, the sample $1_{\text {exc }}$ refers to the event sample obtained after the showering, matching and extra-jet veto of a set of $t \bar{t}+1$-parton PL events. The event sample obtained by combining $0_{e x c}+1_{e x c}+\ldots+(n-1)_{e x c}+n_{i n c}$ will be referred to as the $S_{n}$ sample.

The sample $S_{n}$ constructed according to the above prescriptions can then be used for arbitrary analyses of the final states. The analysis phase is independent of the way the sample was generated; in particular one is allowed to choose an arbitrary jet-finding algorithm $\left(k_{\perp}\right.$, cone, mid-point cone, ...), possibly different than the algorithm used to carry out the matching. It's for a mere matter of convenience that we adopt here the same clustering algorithm that we have used at the matching stage, namely the GETJET jet definition. Jet observables are built out of the partons emerging form the shower in the rapidity range $|\eta| \leq 6$. The jet cone size is set to $R_{\text {cone }}=0.7$ and the minimum transverse momentum to define a jet is $15 \mathrm{GeV}$ at the Tevatron, and $20 \mathrm{GeV}$ at the LHC.

\subsection{Comparison with parton-level results}

In this section we compare inclusive distributions obtained after the shower evolution and matching with the distributions derived at the pure PL. Here we confine ourselves to the case of 0 and 1 light partons, analyzing the results of the $S_{1}=0_{\text {exc }}+1_{\text {inc }}$ combined sample.

The cross-section results obtained for the Tevatron and LHC are shown in table 1. For the results after shower and matching, we quote the individual rates obtained for the $0_{\text {exc }}$ and $1_{\text {inc }}$ samples, together with their sum, which is the only physical quantity. For the PL results, we quote separately the $\mathcal{O}\left(\alpha_{s}^{2}\right)$, Born level cross section, and the full NLO, $\mathcal{O}\left(\alpha_{s}^{3}\right)$, cross section [25]. We notice that the matching algorithm reproduces very well the inclusive LO cross section, where the rate reduction of the $t \bar{t} \mathrm{PL}$ process due to the exclusive veto that removes jet events after the shower is properly compensated by the rate of the $t \bar{t}+1$ parton process (the almost complete agreement in the LHC case should be taken as accidental). 
Figure 1 shows a comparison in absolute rates, for the Tevatron and the LHC, of four inclusive observables evaluated at the PL and after shower evolution. We plot the transverse momentum $\left(p_{T}^{\text {top }}\right)$ and rapidity spectra $\left(y_{t o p}\right)$ of the top quark, , the spectrum of the transverse momentum of the $t \bar{t}$ pair, $p_{T}^{t \bar{t}}$, and the azimuthal correlation $\Delta \phi^{t \bar{t}}$ between the $t$ and $\bar{t}$ quarks. For $p_{T}^{t o p}$ and $y_{t o p}$ we compare the result of the $S_{1}$ sample with the PL Born spectrum. The sub-contribution coming from the $0_{e x c}$ subset is also shown, as a dashed histogram. The $p_{T}^{t \bar{t}}$ and $\Delta \phi^{t \bar{t}}$ distributions are nontrivial only starting at $\mathcal{O}\left(\alpha_{s}^{3}\right)$ and therefore we compare the $S_{1}$ results with the NLO calculation of ref. [25], in which the divergent terms at $p_{T}^{t \bar{t}}=0$ and at $\Delta \phi^{t \bar{t}}=\pi$ are cancelled between the real and virtual contributions.

The agreement for $p_{T}^{t o p}$ is excellent. Likewise, there is excellent agreement for $p_{T}^{t \bar{t}}$ and $\Delta \phi^{t \bar{t}}$ as soon as we move away from the regions dominated by Sudakov effects $\left(p_{T}^{t \bar{t}}=0\right.$ and $\left.\Delta \phi^{t \bar{t}}=\pi\right)$, effects which are incorporated in the $S_{1}$ sample but which are not present in the NLO calculation.

Notice that at the LHC the Sudakov effects are much stronger, as shown in the plot of the $p_{T}^{t \bar{t}}$ variable. The first few empty bins in the NLO result are due to the complete cancellation between the negative virtual rate at $p_{T}^{t \bar{t}}=0$ and the positive $\mathcal{O}\left(\alpha_{s}^{3}\right)$ rate integrated up to approximately $35 \mathrm{GeV}$. Above this threshold, the ALPGEN $S_{1}$ result and the NLO one agree very well. These large Sudakov effects indicate that a fixed-order, $\mathcal{O}\left(\alpha_{s}^{3}\right)$, calculation with parton $p_{T}$ below 40-50 $\mathrm{GeV}$ is not reliable. Nevertheless, in spite of the fact that we generated the 1-parton sample with a threshold of $p_{T}^{\text {min }}=30 \mathrm{GeV}$, the combination of matching and jet veto leads to a smooth interpolation between the soft and hard $p_{T}^{t \bar{t}}$ regions, as will be confirmed in a later section with the comparison with the full NLO+shower treatment of MC@NLO.

These results give us good confidence that the matching algorithm allows to merge the 0-parton and 1-parton samples with the proper removal of double counting, and the accurate description of the hard-jet emission probability. A more complete comparison with a NLO calculation including the Sudakov effects is given below, where we analyze $t \bar{t}$ production using MC@NLO. 

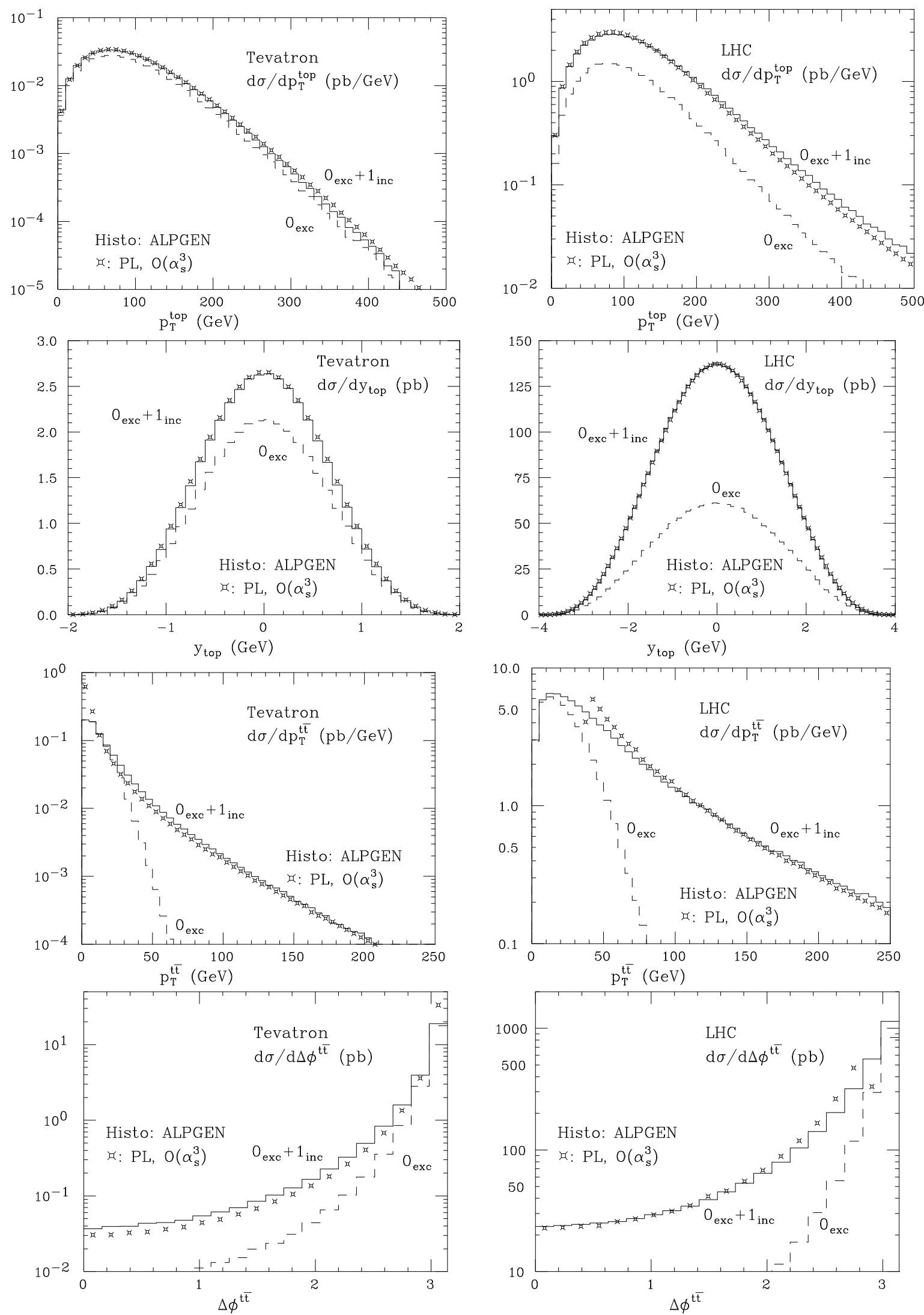

Figure 1: Comparison of the ALPGEN $S_{1}$ results and the LO PL spectra for the inclusive transverse momentum and rapidity of top quarks, for the transverse momentum of the $t \bar{t}$ pair, and for their azimuthal correlations. All distributions are absolutely normalized. The contribution of the $0_{e x c}$ sample is shown by the dashed line. The plots on the left are for the Tevatron, those on the right for the LHC. 


\begin{tabular}{|l|lr|}
\hline & Tevatron & LHC \\
\hline $0_{\text {exc }}$ & 3.42 & 216.6 \\
$1_{\text {exc }}$ & 0.66 & 149.9 \\
$2_{\text {exc }}$ & 0.09 & 65.8 \\
$3_{\text {inc }}$ & 0.010 & 29.9 \\
\hline Total & 4.18 & 462.2 \\
\hline
\end{tabular}

Table 2: Cross sections (in pb), for $t \bar{t}$ production at the Tevatron and LHC. The contribution of the different parton samples, for the default generation and matching options. The relative numerical integration precision is at the permille level.

\subsection{Impact of higher-order parton processes}

In this section we introduce higher-multiplicity final states in the matrix element (ME) calculation. In particular, we generate PL samples with up to 3 final-state partons in addition to the $t \bar{t}$ pair. After showering and matching, these events are combined into the fully inclusive $S_{3}$ sample, contributing with the cross-sections given in table 2 . Notice that the overall rates are well consistent with those obtained with the $S_{1}$ sample, in table 1 . This indicates that the matching algorithm correctly ensures that the 1-parton inclusive rate, $\sigma\left(1_{\text {inc }}\right)$, is reproduced by the sum of the partial contributions, $\sigma\left(1_{\text {exc }}\right)+\sigma\left(2_{\text {exc }}\right)+\sigma\left(3_{\text {inc }}\right)$.

This consistency is maintained, at the level of spectra, for the inclusive distributions that receive their LO contributon from the 0- and 1-parton final states. This is shown in the first three plots of fig. 2, where we compare the predictions for $p_{T}^{t o p}, p_{T}^{t \bar{t}}, \Delta \phi^{t \bar{t}}$ and $p_{T, 1}$ (the leading-jet $p_{T}$ ) obtained, for the Tevatron, with the $S_{1}$ and $S_{3}$ event samples. In the figures we also display the incremental contribution given by the various subsets, $0_{e x c}, 1_{e x c}$ and $2_{e x c}$. The lower insets represent the relative difference between the two results, $\left[d \sigma\left(S_{1}\right)-d \sigma\left(S_{3}\right)\right] / d \sigma\left(S_{3}\right)$. As one can see these differences are at the few-\% level at most, except in the high-momentum tail of the $p_{T}$ distributions at the LHC, where the $S_{3}$ spectrum is harder than $S_{1}$.

More interesting is the case of observables that receive their LO contribution from final states with more than 1 extra hard parton. The comparison between the predictions of the $S_{1}$ and $S_{3}$ samples can tell us more about possible limitations of the shower MC in describing hard emissions leading to extra jets. We start by plotting the jet multiplicity distribution, $N_{\text {jet }}$, in the upper panels of fig. 3. The agreement between the two calculations is remarkable, at the level of better than $20 \%$ even for the large jet multiplicities. This can be justified by the fact that the $20 \mathrm{GeV}$ jets we are considering here are rather soft objects when compared with the total amount of energy involved in a $t \bar{t}$ event. Therefore the soft-approximation used in the shower evolution correctly describes the emission rate of these multijet events. This is confirmed by the plots in the second row, showing the $p_{T}$ spectrum of the 2nd leading jet in the event. Once again the agreement between the two calculations is excellent, up to very large values of the jet $E_{T}$. Where the shower approximation appears to be less reliable is in the description of the kinematical correlations between the jets. The lower plots of the figure show the $(\eta, \phi)$ correlations between the 1st and 2 nd and between the 2nd and 3rd jets. Clear differences in the shapes are evident. 

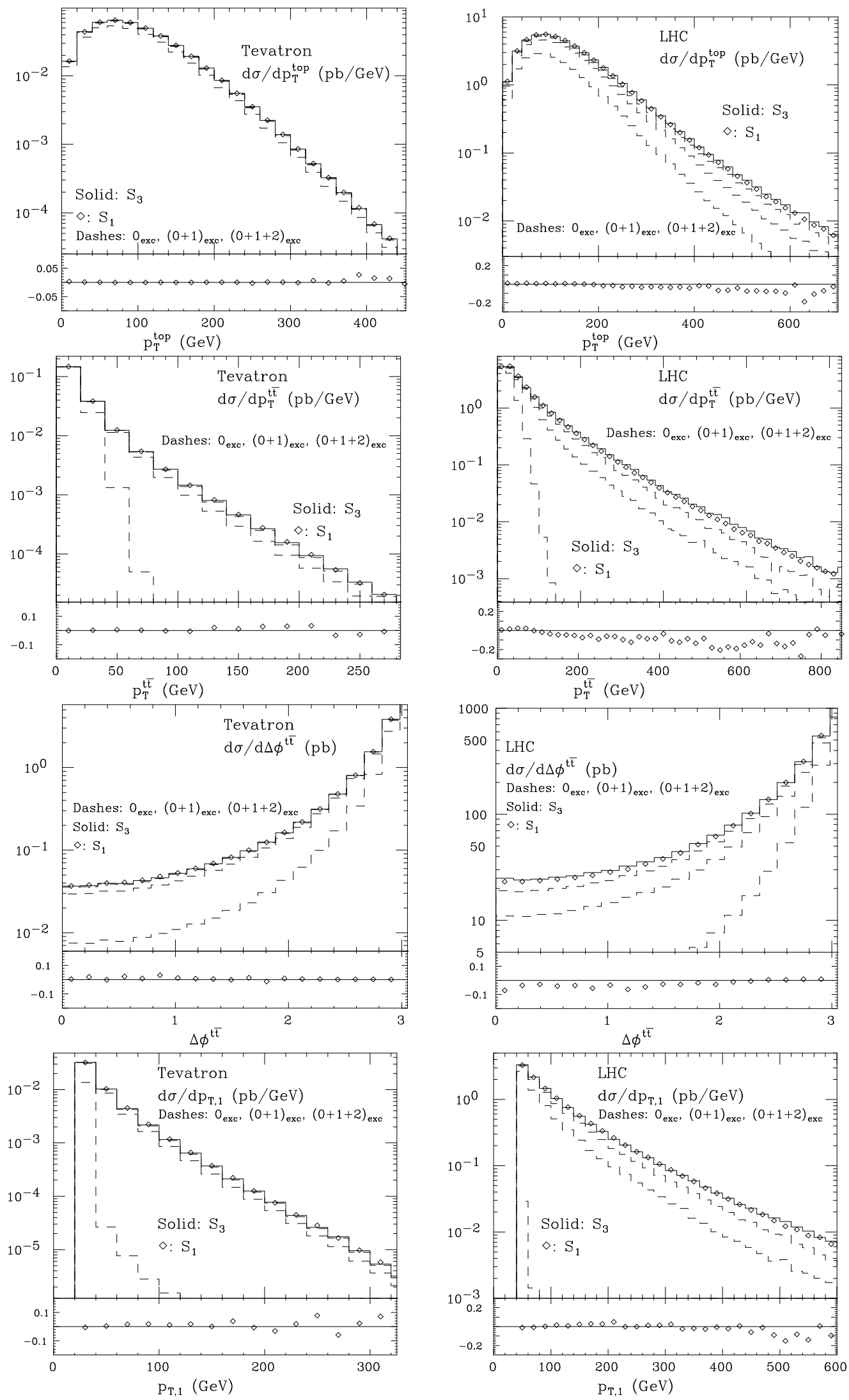

Figure 2: Comparison between the distributions obtained from the $S_{1}$ event samples $\left(0_{e x c}+1_{\text {inc }}\right)$ and from the $S_{3}$ event samples $\left(0_{e x c}+1_{e x c}+2_{e x c}+3_{i n c}\right)$, for various $(\leq 1)$-parton observables at the Tevatron (left-hand side) and LHC (right-hand side). Cumulative contributions from the $0_{e x c}, 1_{e x c}$ and $2_{e x c}$ subsamples are shown by the dashed histograms. 

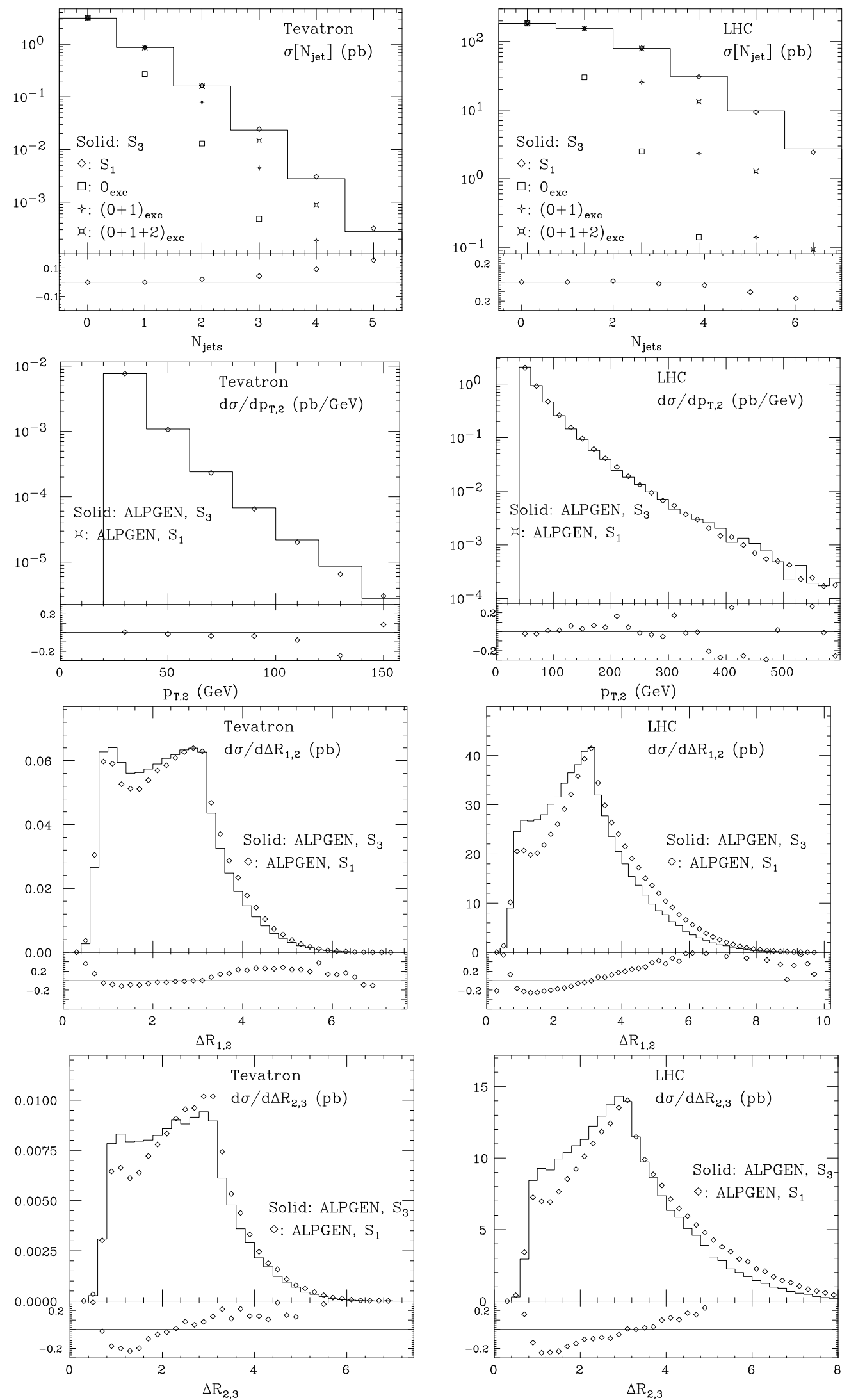

Figure 3: Comparison between the distributions obtained from the $S_{1}$ event samples $\left(0_{e x c}+1_{\text {inc }}\right)$ and from the $S_{3}$ event samples $\left(0_{e x c}+1_{e x c}+2_{e x c}+3_{i n c}\right)$, for various higher-order parton observables at the Tevatron (left-hand side) and at the LHC (right-hand side). 


\subsection{Study of the generation and matching systematics}

In this section we explore the systematic uncertainties due to the variation of generation and matching parameters. These uncertainties reflect the underlying fact that this approach relies on the LO evaluation of the hard ME and on the LL accuracy in the removal of double counting and in the description of the shower evolution. As mentioned in the beginning, the ultimate goal of this approach is to enable the generation of fully inclusive event samples that offer LL accuracy throughout phase-space, including configurations with many jets. In this section we shall show that the size of the resulting uncertainties is consistent with what can be expected in such a LL approach in the case of $t \bar{t}$ production. A more thorough discussion of matching systematics can be found in ref. [10].

It should be remarked that the presence in this approach of extra parameters - such as the matching parameters - compared to the usual PL or shower-only approaches is not necessarily a curse. The ultimate use of LL event generators is not to incorporate and enable high-precision predictions of QCD, but rather to provide the most faithful representation of the data, so that the experimental searches for and studies of new phenomena can be built on a solid foundation. The uncertainties introduced by the possibility to change the matching and generation parameters should therefore be seen as an opportunity to optimize, via their fitting, the agreement between the generator and the data.

In our examples here we consider two independent variations of the generation and of two of the matching cuts, keeping fixed our definition of the physical objects (the jets) and of the observables. For the generation variations we maintain the relation between generation and matching cuts given in eq. (3.1), and consider a lowering and an increase of the $p_{T}^{\text {min }}$ thresholds. For the matching variations we keep fixed the generation parameters, and consider a change in the $E_{T}$ threshold for the clusters, $E_{T}^{\text {clus }}$, and a change in the minimal separation $R_{\text {match }}$ required for a parton and a cluster to match. The numerical values are detailed in table 3 .

We start by discussing the cross sections, which are given in tables 4 and 5 . While the contributions of the individual partonic samples can vary by a large amount, the total cross sections are very stable, with the maximum excursion between minimum and maximum being of the order of $5 \%$. For comparison, the rate of the $0_{e x c}$ samples, which are the dominant ones, can vary by up to $35 \%$.

Then we proceed to study some distributions, following the template of the comparisons between the $S_{1}$ and $S_{3}$ samples discussed earlier. For the Tevatron, the observables dominated by contributions with up to 1 hard parton are shown in fig. 4 , and those relative to multijet final states in fig. 5. Even the rates for large jet multiplicities are extremely stable. Consider for example the 3 -jet bin. At the Tevatron, the contribution of the $3_{\text {inc }}$ sample varies from $10 \mathrm{fb}$, for the default generation/matching cuts, to $24 \mathrm{fb}$ for set $\mathrm{G} 1$ and $2 \mathrm{fb}$ for set G2. Nevertheless the total 3-jet rates show a stability at the level of $10 \%$, as do the shapes of the distributions (see for example the $\Delta R_{2,3}$ case in the figure).

The analysis at the LHC leads to qualitatively and quantitatively similar results, which will not be shown here. 


\begin{tabular}{|l|cc|cc|}
\hline & \multicolumn{2}{|c|}{ Generation parameters } & \multicolumn{2}{c|}{ Matching parameters } \\
Param set & $p_{T}^{\text {min }}$ & $R_{\text {min }}$ & $\min E_{T}^{\text {clus }}$ & $R_{\text {match }}$ \\
\hline Tevatron, default & 20 & 0.7 & 25 & $1.5 \times 0.7$ \\
Tevatron, Set G1 & 15 & 0.7 & 20 & $1.5 \times 0.7$ \\
Tevatron, Set G2 & 30 & 0.7 & 36 & $1.5 \times 0.7$ \\
Tevatron, Set M1 & 20 & 0.7 & 20 & $1.5 \times 0.7$ \\
Tevatron, Set M2 & 20 & 0.7 & 25 & $1.5 \times 1.0$ \\
\hline LHC, default & 30 & 0.7 & 36 & $1.5 \times 0.7$ \\
LHC, Set G1 & 25 & 0.7 & 30 & $1.5 \times 0.7$ \\
LHC, Set G2 & 40 & 0.7 & 48 & $1.5 \times 0.7$ \\
LHC, Set M1 & 30 & 0.7 & 30 & $1.5 \times 0.7$ \\
LHC, Set M2 & 30 & 0.7 & 36 & $1.5 \times 1.0$ \\
\hline
\end{tabular}

Table 3: Variations of the generation and matching parameters used for the study of the systematics.

\begin{tabular}{|l|l|l|l|l|l|}
\hline Tevatron & Default & Set G1 & Set G2 & Set M1 & Set M2 \\
\hline $0_{\text {exc }}$ & 3.42 & 3.15 & 3.79 & 3.14 & 3.33 \\
$1_{\text {exc }}$ & 0.66 & 0.82 & 0.42 & 0.78 & 0.74 \\
$2_{\text {exc }}$ & 0.09 & 0.15 & 0.036 & 0.13 & 0.11 \\
$3_{\text {inc }}$ & 0.010 & 0.024 & 0.002 & 0.021 & 0.012 \\
\hline Total & 4.18 & 4.14 & 4.25 & 4.08 & 4.19 \\
\hline
\end{tabular}

Table 4: Cross sections (in pb), for $t \bar{t}$ production at the Tevatron. The contribution of the different parton samples, for various generation and matching options. The columns are labeled according to the parameter definitions introduced in table 3 . The relative numerical integration precision is at the permille level.

\begin{tabular}{|l|l|l|l|l|l|}
\hline LHC & Default & Set G1 & Set G2 & Set M1 & Set M2 \\
\hline $0_{\text {exc }}$ & 217 & 185 & 267 & 185 & 203 \\
$1_{\text {exc }}$ & 150 & 156 & 134 & 148 & 160 \\
$2_{\text {exc }}$ & 66 & 81 & 44 & 74 & 76 \\
$3_{\text {inc }}$ & 30 & 45 & 15 & 40 & 35 \\
\hline Total & 462 & 467 & 460 & 447 & 475 \\
\hline
\end{tabular}

Table 5: Same as table 4, for the LHC. 

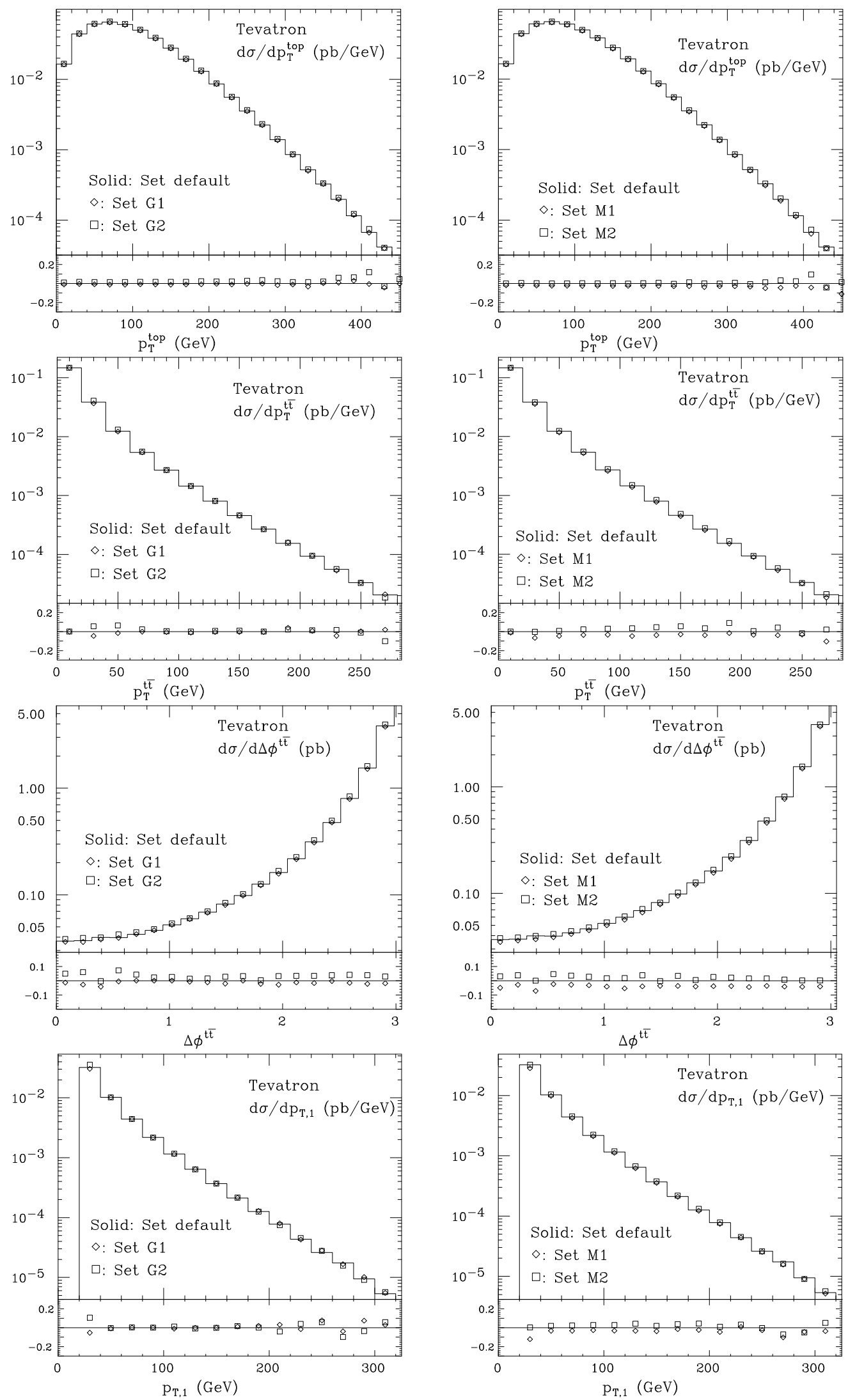

Figure 4: Comparison between the three alternative sets of generation (left) and matching (right) parameters given in table 3 , at the Tevatron. 

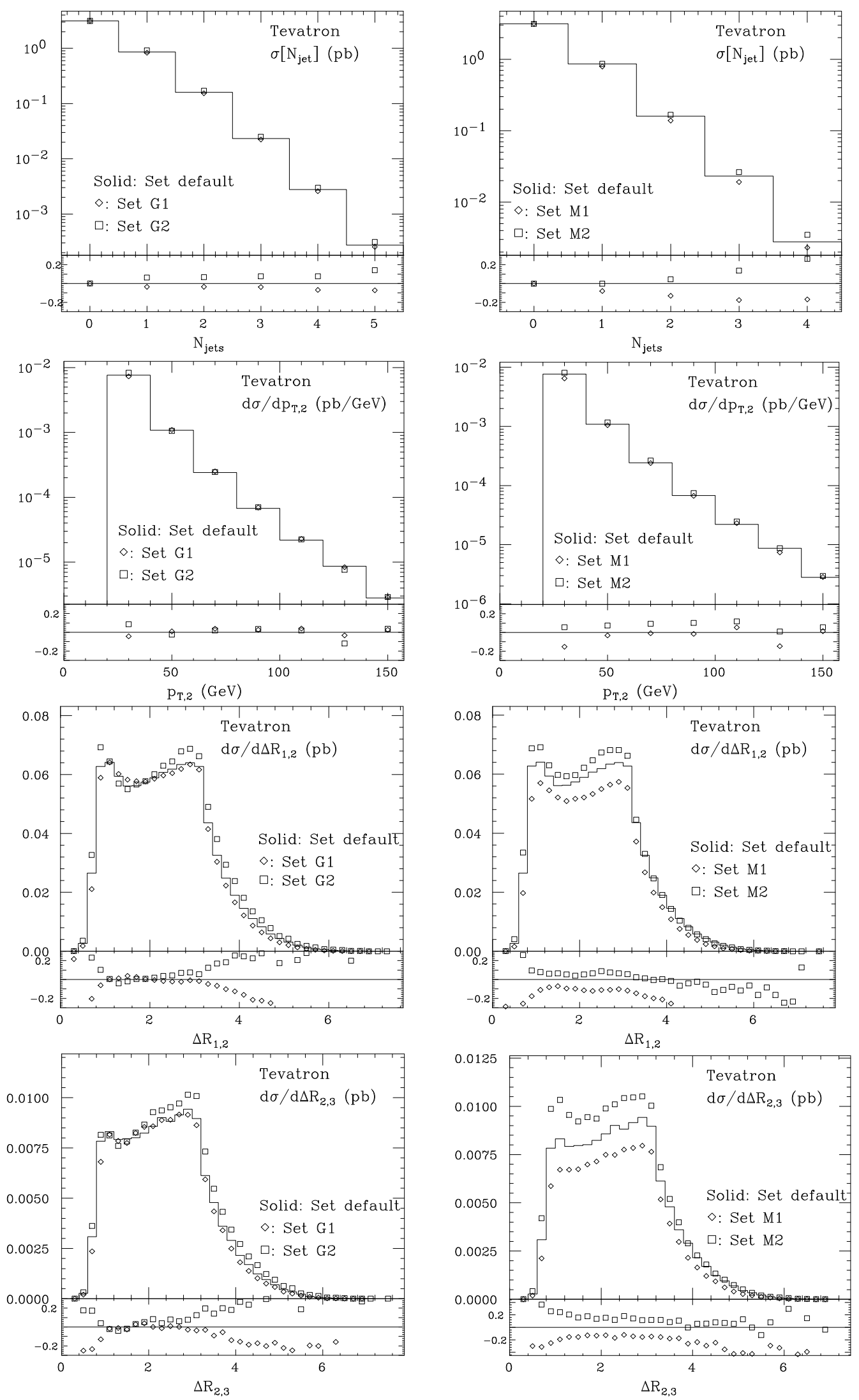

Figure 5: Comparison between the three alternative sets of generation and matching parameters given in table 3 , for multijet distributions at the Tevatron. 


\section{Comparisons with MC@NLO}

We shall now compare in detail the description of $t \bar{t}$ events as provided by ALPGEN and MC@NLO. For consistency with the MC@NLO approach, where only the $\mathcal{O}\left(\alpha_{s}^{3}\right)$ ME effects are included, we use ALPGEN samples obtained summing the $0_{e x c}$ and $1_{i n c}$ contributions. As in the case of the results shown before, all plotted quantities refer to $t$ and $\bar{t}$ quarks regarded as stable and after performing the showering of the event but without including any non perturbative effect (non perturbative gluon splitting, hadronization, underlying event,...).

To match MC@NLO's default we have used, for both codes, the same factorization and renormalization scale

$$
\mu^{2}=\frac{1}{2}\left(p_{t, T}^{2}+m_{t o p}^{2}+p_{\bar{t}, T}^{2}+m_{t o p}^{2}\right)
$$

the same set of PDF MRST2001J[23] and the same value for the top mass (175 GeV).

The upper two rows of plots in figs. 6 and 7 refer to inclusive properties of the $t \bar{t}$ system, namely the transverse momentum and rapidity of the top and anti-top quark, the transverse momentum of the $t \bar{t}$ pair, and the azimuthal angle $\Delta \phi^{t \bar{t}}$ between the top and anti-top quark. The overall agreement is good, once ALPGEN is corrected with the proper K-factor (1.36 for the Tevatron, and 1.51 for the LHC), and no large discrepancy is seen between the two descriptions of the chosen distributions. The most significant differencies (10 to $20 \%$ ) are seen in the $p_{T}^{\text {top }}$ distribution, ALPGEN's one being slightly softer.

The study of jet quantities reveals instead one important difference: the rapidity of the leading jet, $y_{1}$, is different in the two descriptions, where MC@NLO exhibits a dip at $y_{1}=0$. This difference is particularly marked at the Tevatron, but is very visible also at the LHC. This is shown in the right figure of the third row in figs. 6 and 7 .

Furthermore, while the $p_{T}$ spectrum of the 2nd jet is rather similar in the two approaches at the Tevatron, the agreement becomes worse at the LHC. Visible differences are also present in the distribution of the 1 st and 2 nd jet separation in $(\eta, \phi)$ space, $\Delta R_{1,2}$.

Figure 8, finally, shows the comparison of the jet multiplicity distributions. 

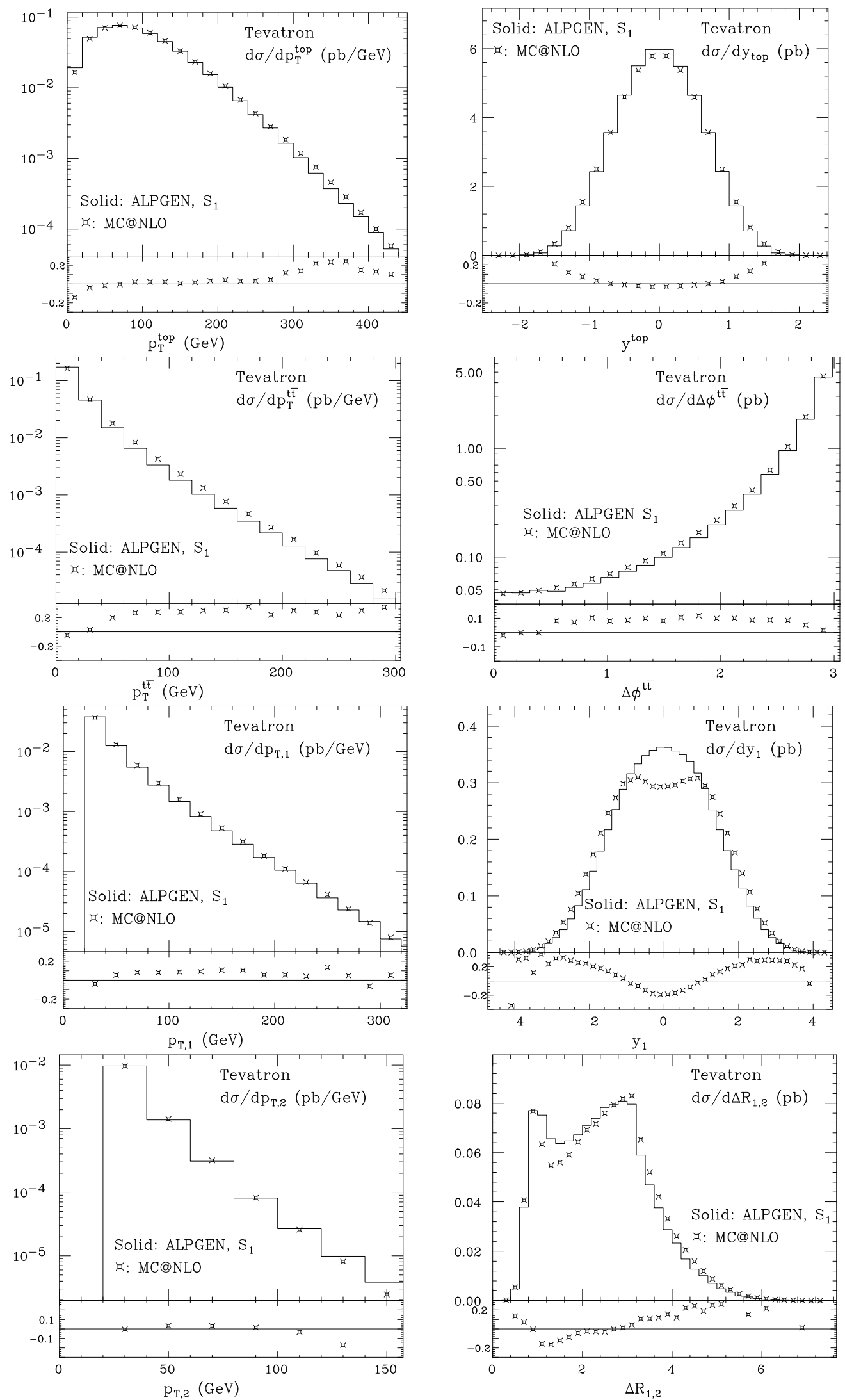

Figure 6: Comparison of ALPGEN (histogram) and MC@NLO (plot) distributions, at the Tevatron. The ALPGEN results are rescaled to MC@NLO, using the K factor of 1.36. The relative difference (MC@NLO-ALPGEN)/ALPGEN) is shown at the bottom of each plot. 

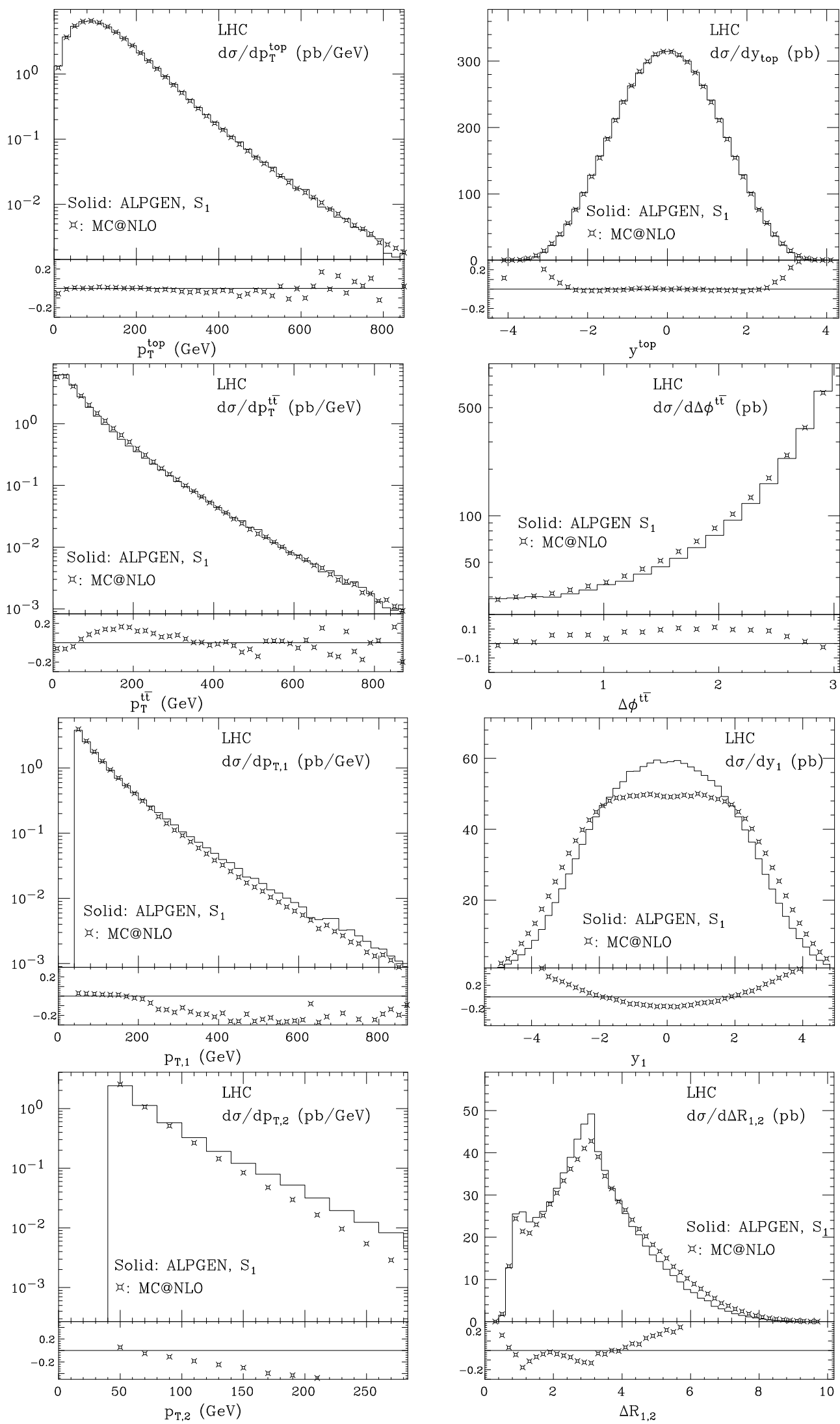

Figure 7: Same as fig. 6 for the LHC, using the K factor of 1.51 . 

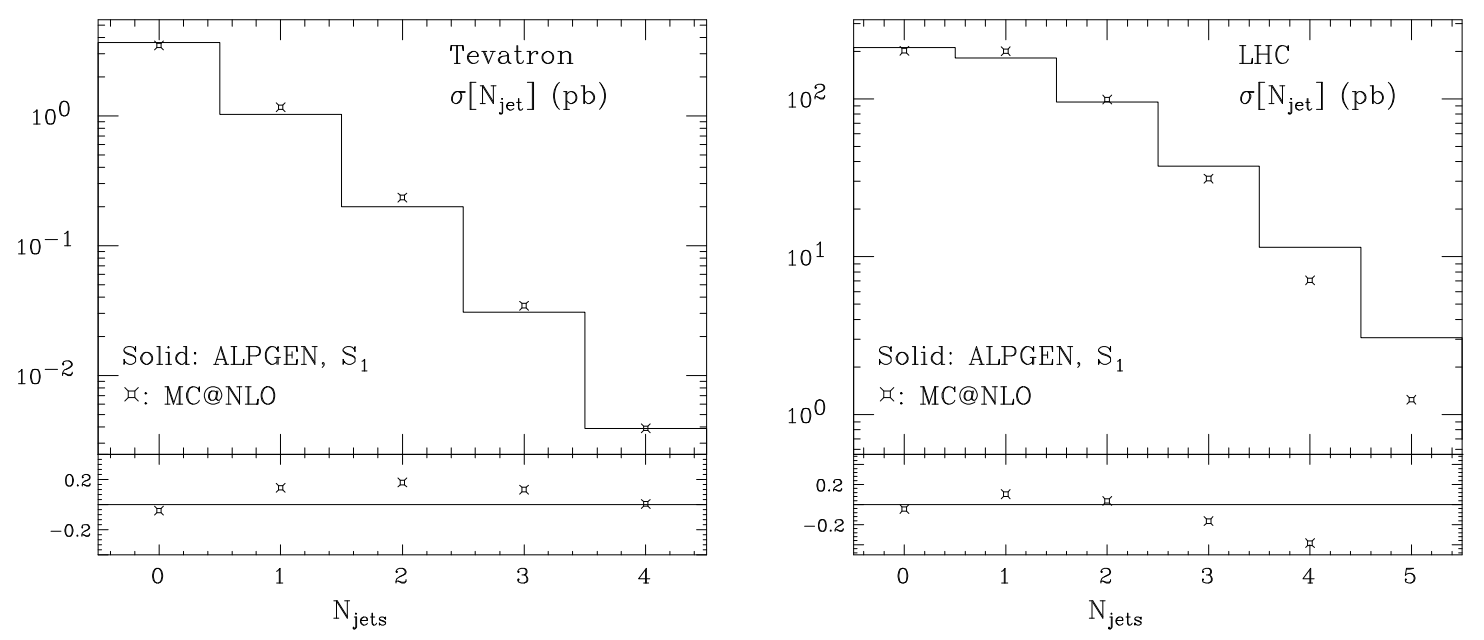

Figure 8: Jet multiplicity from ALPGEN and MC@NLO, at the Tevatron (left) and at the LHC (right). The relative difference (MC@NLO-ALPGEN)/ALPGEN is shown at the bottom of each plot.
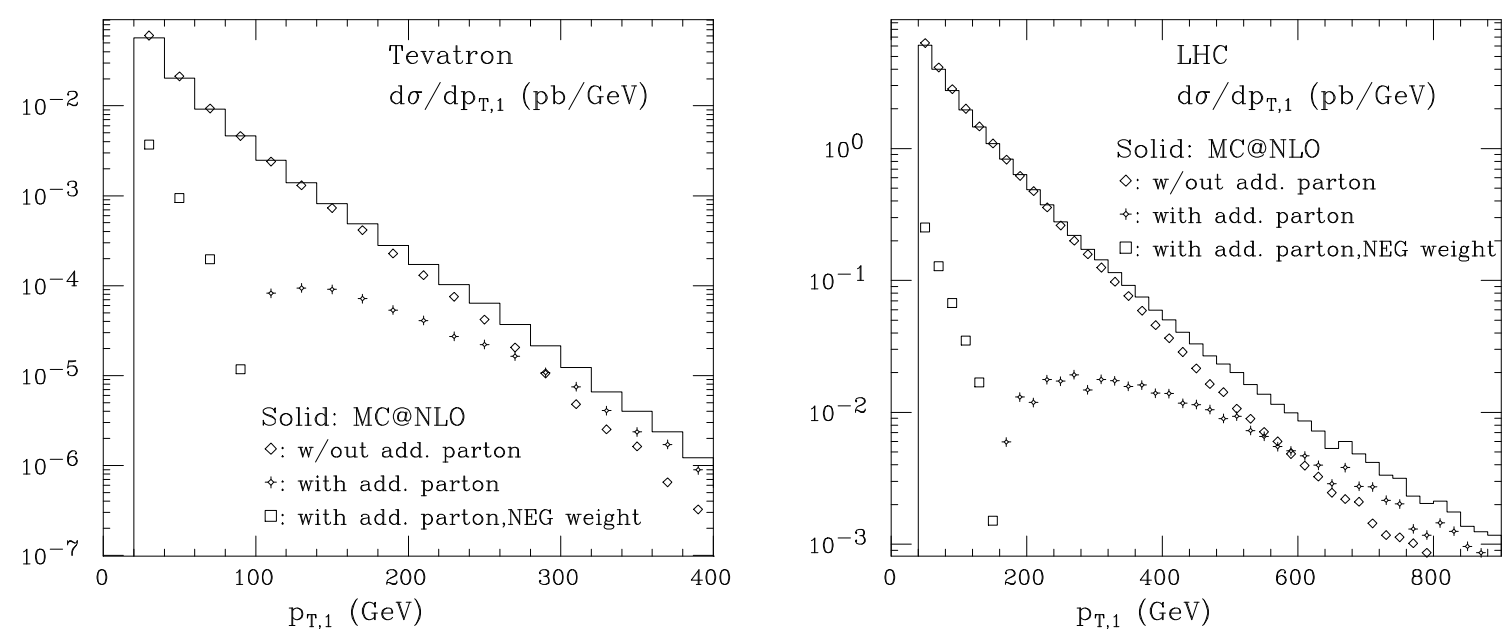

Figure 9: Contributions to the transverse momentum of the leading jet in MC@NLO. Tevatron (left) and LHC (right).

To understand the difference in the rapidity distribution, we look in more detail in fig. 9 at some features in the MC@NLO description of the leading jet. For the $p_{T}$ of the leading jet, $p_{T, 1}$, we plot separately the contribution from the various components of the MC@NLO generation: events in which the shower is initiated by the LO $t \bar{t}$ hard process, and events in which the shower is initiated by a $t \bar{t}+q(g)$ hard process. In this last case, we separate the contribution of positive- and negative-weight events, where the distribution of negative events is shown in absolute value. The plots show that for MC@NLO the contribution of the $t \bar{t}+q(g)$ hard process is almost negligible over most of the relevant range and becomes appreciable only for very large values of $p_{T, 1}$. This hierarchy is stronger at the LHC than at the Tevatron.

Figure. 10 shows the various contributions to the rapidity distribution $y_{1}$ for different jet $p_{T}$ thresholds. It appears that the $y_{1}$ distribution resulting from the shower evolution of the $t \bar{t}$ events in $\mathrm{MC} @ \mathrm{NLO}$ has a strong dip at $y_{1}=0$, a dip that cannot be compensated by the more central distributions of the jet from the $t \bar{t}+q(g)$ hard process, given its marginal role in the overall jet rate. 

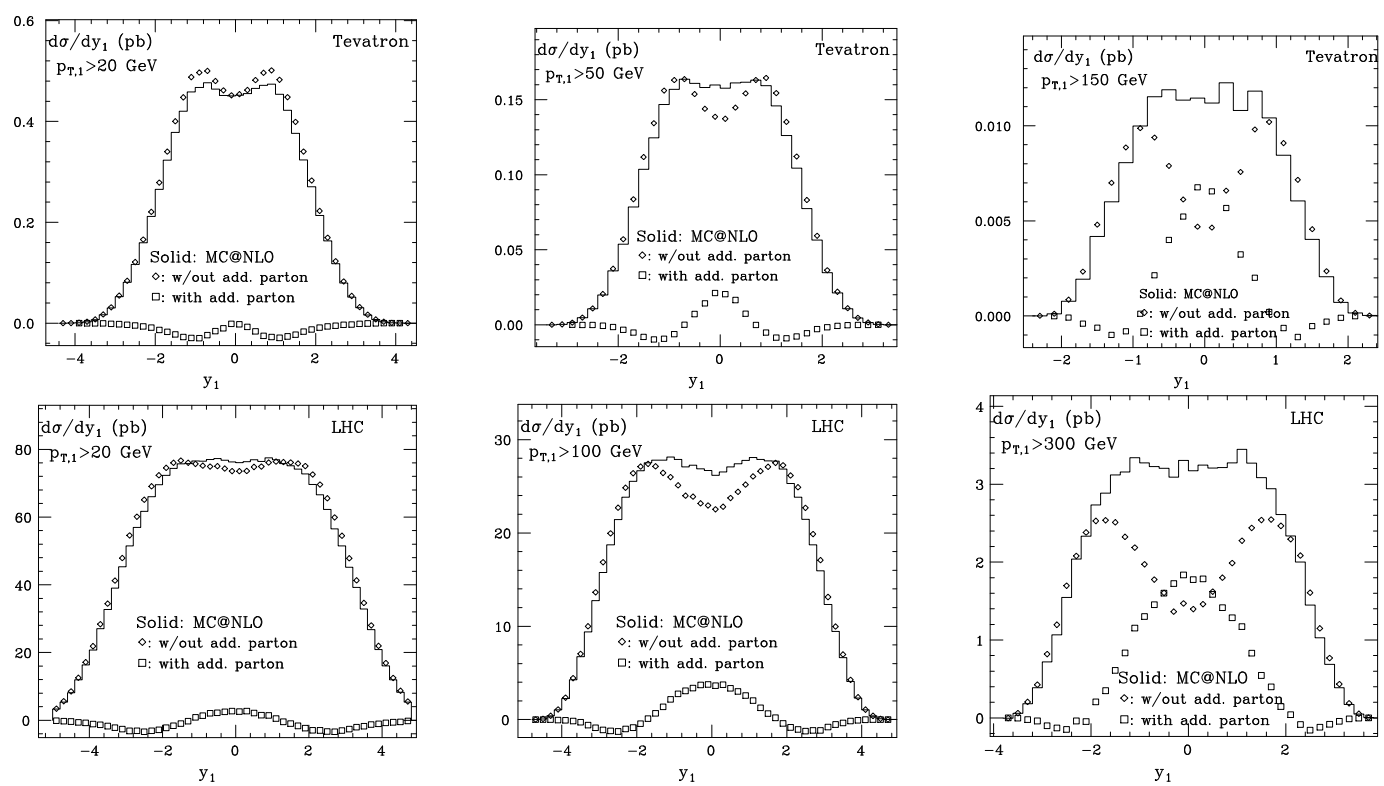

Figure 10: Rapidity of the leading jet $y_{1}$ as described by MC@NLO. The plots show the results for various jet $p_{T}$ thresholds. Upper set: Tevatron, lower set: LHC

That the dip at $y_{1}=0$ is a feature typical of jet emission from the $t \bar{t}$ state in HERWIG is shown in fig. 11, obtained from the standard HERWIG code rather than from MC@NLO. We speculate that this feature is a consequence of the dead-cone description of hard emission from heavy quarks implemented in the HERWIG shower algorithm. To complete our analysis, we show in fig. 12 the comparison between the ALPGEN, MC@NLO and the parton-level $y_{1}$ spectra, for different jet $p_{T}$ thresholds. We notice that at large $p_{T}$, where the Sudakov effects that induce potential differences between the shower and the PL results have vanished, the ALPGEN result reproduces well the PL result, while still differing significantly from the MC@NLO distributions. 

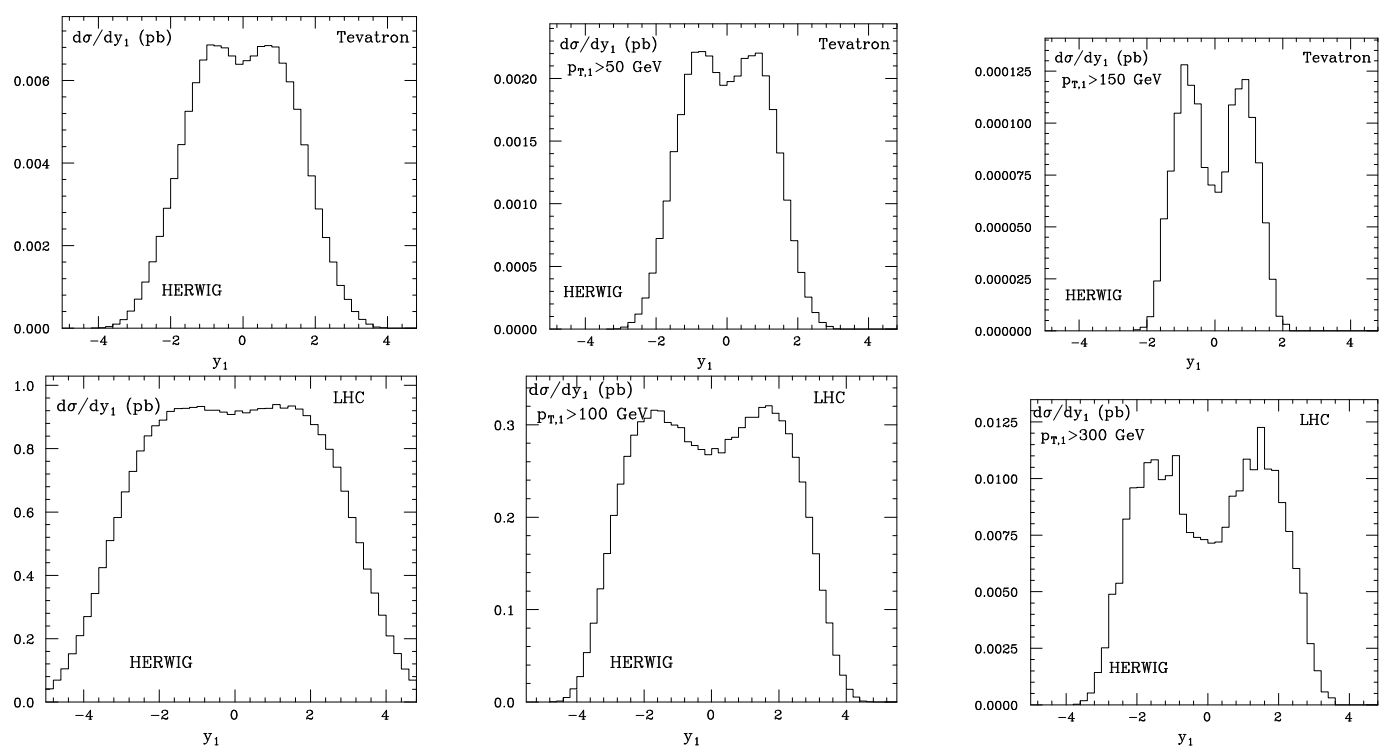

Figure 11: Rapidity of the leading jet $y_{1}$ as described by HERWIG. The plots show the results for various jet $p_{T}$ thresholds. Upper set: Tevatron, lower set: LHC
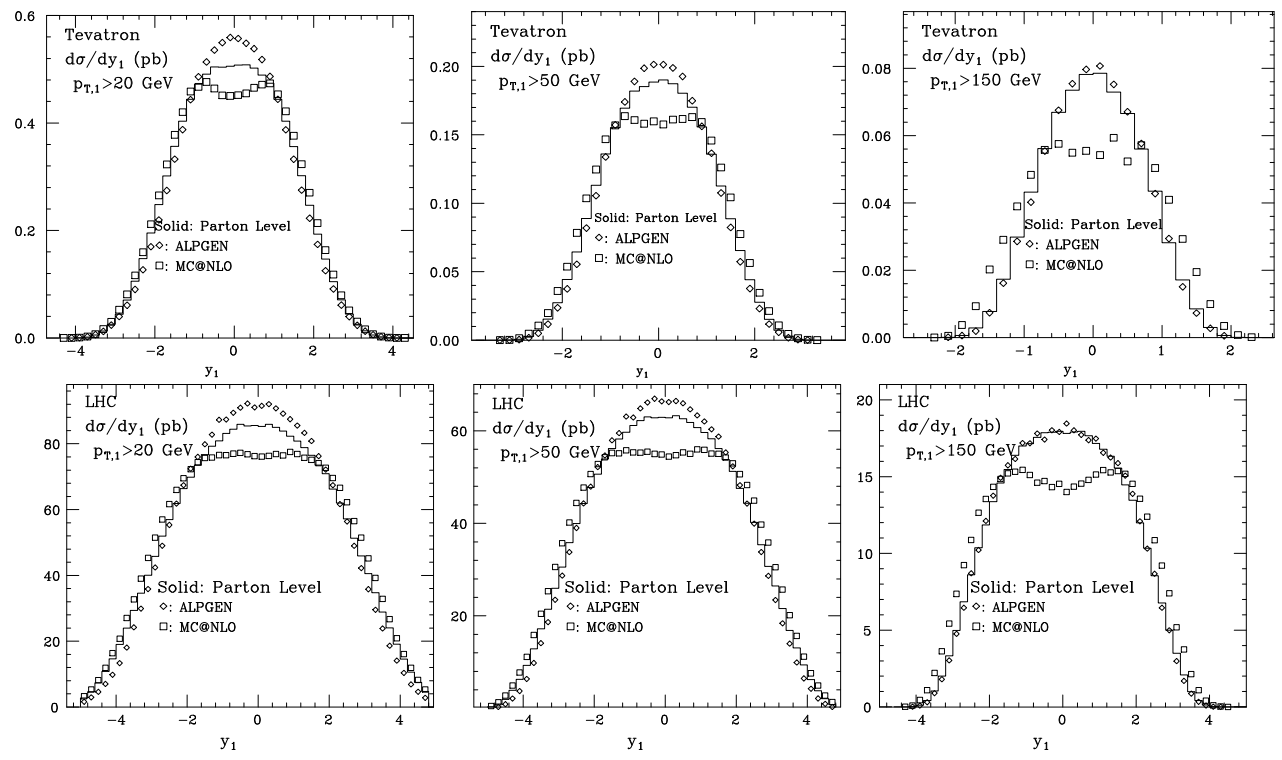

Figure 12: Rapidity spectrum of the leading jet, as predicted by ALPGEN, MC@NLO, and by the parton level, for various $p_{T}$ thresholds of the jet. Upper curves: Tevatron; lower curves: LHC. 


\subsection{Spin correlations in top decays}

Top decays are described differently in the two codes. In MC@NLO the top quark is assumed stable at the parton level and it is then HERWIG that models the decay: production and decay are thus uncorrelated, and spin correlations are missing. In ALPGEN, on the other hand, spin correlations are taken into account in the evaluation of the matrix elements, and the proper correlations are then preserved by the shower evolution. This is a minor issue for MC@NLO, which is being addressed in its forthcoming releases ${ }^{1}$. We show here nevertheless a study of the impact of spin correlations, to conclude that indeed it is important to keep track of them for a reliable simulation of the final state kinematics.

To this end we have selected the leptonic decay channel for both top and antitop, and studied, after showering, several dilepton distributions. For simplicity we just present the results for the Tevatron, since those for the LHC exhibit the same features. In fig. 13 we plot the transverse momentum $p_{T}^{\text {lept }}$ and the rapidity $y^{\text {lept }}$ of the leading lepton, the invariant dilepton mass and the azimuthal difference $\Delta \phi^{\text {lept }}$ between the two leptons in the tranverse plane. For ALPGEN we plot the distribution with and without spin correlation taken into account. The angular separation $\Delta \phi^{\text {lept }}$ and the invariant charged dilepton mass exhibit some sensitivity to spin correlation which is more evident at higher energies. The other quantities look fairly insensitive to spin correlations. Notice that, as expected, MC@NLO behaves exactly like ALPGEN without spin correlations.
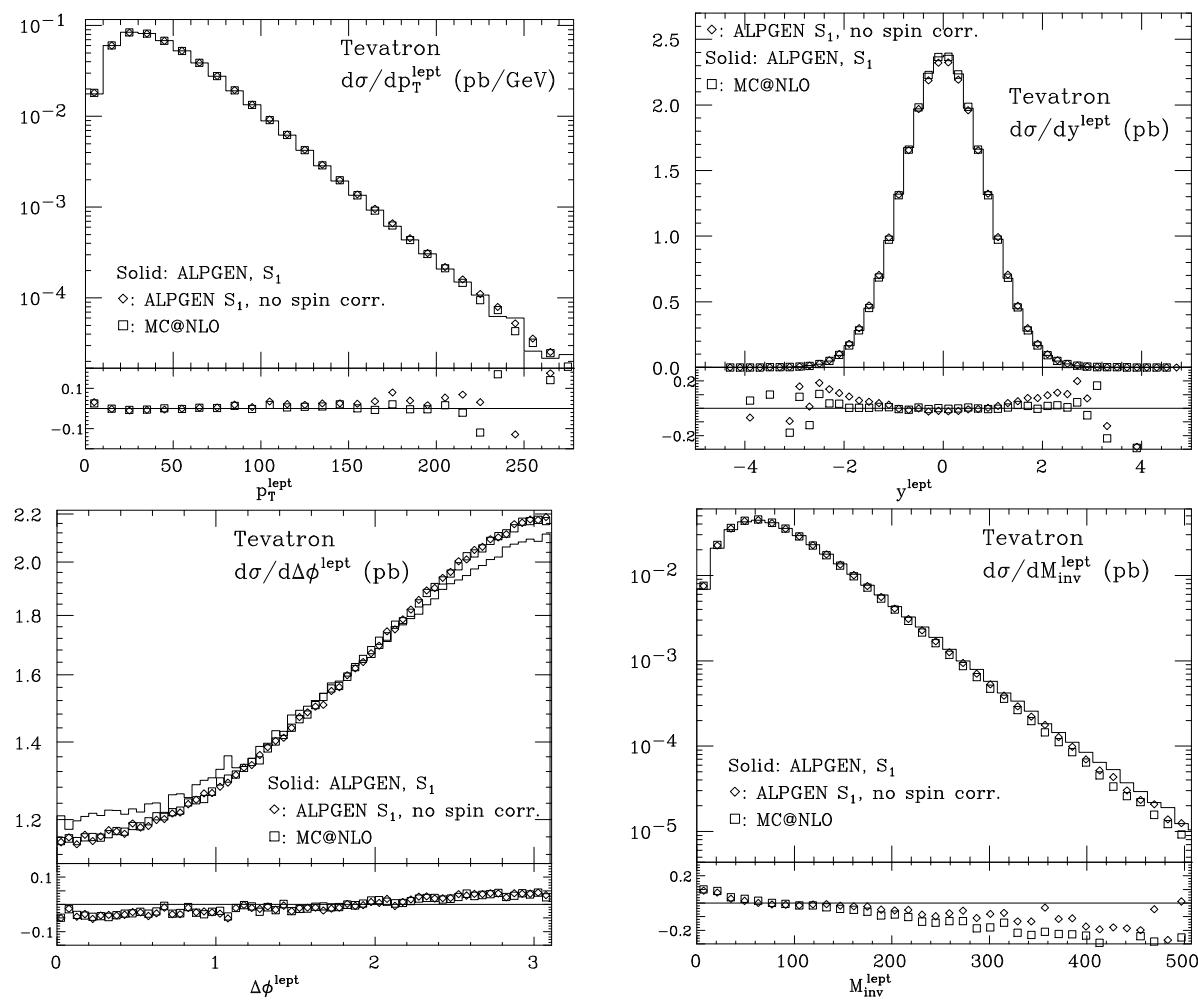

Figure 13: Leptonic distributions for the default ALPGEN, for ALPGEN without spin correlations in top decays, and for MC@NLO.

\footnotetext{
${ }^{1}$ S. Frixione, private communication.
} 


\section{Conclusions}

The study presented in this paper examines the predictions of ALPGEN and its matching algorithm for the description of $t \bar{t}+$ jets events. Several checks of the algorithm have shown its internal consistency, and indicate a rather mild dependence of the results on the parameters that define it. The consistency of the approach is confirmed by the comparison with MC@NLO. In particular, inclusive variables sensitive to the matching at the transition between the $\mathcal{O}\left(\alpha_{s}^{2}\right)$ and $\mathcal{O}\left(\alpha_{s}^{3}\right)$ matrix elements (such as the transverse momentum of the $t \bar{t}$ pair) show excellent agreement, once the $\mathrm{NLO} / \mathrm{LO} K$ factor is included.

We found, on the other hand, a rather surprising difference between the predictions of two codes for the rapidity distribution of the leading jet accompanying the $t \bar{t}$ pair. At large $p_{T}$ one expects the jet spectrum to agree with the $\mathrm{LO}, \mathcal{O}\left(\alpha_{s}^{3}\right)$, parton level calculation. This agreement is verified in the ALPGEN calculation, but is not present in the case of MC@NLO. In view of the relevance of this variable for the study at the LHC of new physics signals including jets in association with top quark pairs (such as $t \bar{t} H$ ), it is important to further pursue the origin of this discrepancy, with independent calculations, and with a direct comparison with data. Preliminary results [17] obtained with the new positive-weight NLO shower MC introduced in [4, 14], appear to support the distributions predicted by ALPGEN. It would also be very interesting to verify whether the Tevatron statistics is sufficient to directly probe this observable, and conclusively resolve this issue.

\section{Acknowledgements}

M. Moretti, F. Piccinini and M. Treccani wish to thank the CERN Theory unit for its kind hospitality and support.

\section{References}

[1] M. L. Mangano and T. J. Stelzer, Ann. Rev. Nucl. Part. Sci. 55 (2005) 555.

[2] M. A. Dobbs et al., arXiv:hep-ph/0403045.

[3] S. Frixione and B. R. Webber, JHEP 0206 (2002) 029 [arXiv:hep-ph/0204244].

[4] P. Nason, JHEP 0411 (2004) 040 [arXiv:hep-ph/0409146].

[5] R. Kuhn, A. Schalicke, F. Krauss and G. Soff, arXiv:hep-ph/0012025.

[6] S. Catani, F. Krauss, R. Kuhn and B. R. Webber, JHEP 0111 (2001) 063 [arXiv:hep-ph/0109231].

[7] L. Lonnblad, JHEP 0205 (2002) 046 [arXiv:hep-ph/0112284].

[8] F. Krauss, JHEP 0208 (2002) 015 [arXiv:hep-ph/0205283].

[9] M.L. Mangano, presentation at the FNAL Matrix Element/Monte Carlo Tuning Working Group, 15 Nov 2002, http://www-cpd.fnal.gov/personal/mrenna/tuning/nov2002/mlm.pdf .

[10] M.L. Mangano, to appear.

[11] S. Mrenna and P. Richardson, JHEP 0405 (2004) 040 [arXiv:hep-ph/0312274].

[12] N. Lavesson and L. Lonnblad, JHEP 0507 (2005) 054 [arXiv:hep-ph/0503293].

[13] S. Hoche, F. Krauss, N. Lavesson, L. Lonnblad, M. Mangano, A. Schalicke and S. Schumann, arXiv:hep-ph/0602031.

[14] P. Nason and G. Ridolfi, JHEP 0608 (2006) 077 [arXiv:hep-ph/0606275]. 
[15] S. Frixione, P. Nason and B. R. Webber, JHEP 0308 (2003) 007 [arXiv:hep-ph/0305252].

[16] S. Frixione and B. R. Webber, arXiv:hep-ph/0309186.

[17] P. Nason, presented at the 3rd meeting of the "Workshop sui Monte Carlo, la fisica e le simulazioni a LHC", Frascati, 23-25 October 2006, http://moby.mib.infn.it/ñason/mcws3/Nason-10-2006.pdf.

[18] S. Frixione, E. Laenen, P. Motylinski and B. R. Webber, JHEP 0603 (2006) 092 [arXiv:hep-ph/0512250].

[19] M. L. Mangano, M. Moretti, F. Piccinini, R. Pittau and A. D. Polosa, JHEP 0307 (2003) 001 [arXiv:hep-ph/0206293];

F. Caravaglios, M. L. Mangano, M. Moretti and R. Pittau, Nucl. Phys. B 539 (1999) 215 [hep-ph/9807570].

[20] G. Marchesini and B. R. Webber, Nucl. Phys. B 310 (1988) 461. G. Marchesini, B. R. Webber, G. Abbiendi, I. G. Knowles, M. H. Seymour and L. Stanco, Comput. Phys. Commun. 67 (1992) 465. G. Corcella et al., JHEP 0101 (2001) 010 [hep-ph/0011363].

[21] T. Sjostrand, Comput. Phys. Commun. 82 (1994) 74. T. Sjostrand, P. Eden, C. Friberg, L. Lonnblad, G. Miu, S. Mrenna and E. Norrbin, Comput. Phys. Commun. 135 (2001) 238 [hep-ph/0010017].

[22] G. Corcella et al., arXiv:hep-ph/0210213.

[23] A. D. Martin, R. G. Roberts, W. J. Stirling and R. S. Thorne, Eur. Phys. J. C 23 (2002) 73 [arXiv:hep-ph/0110215].

[24] F.E. Paige and S.D. Protopopescu, in Physics of the SSC, Snowmass, 1986, Colorado, edited by R. Donaldson and J. Marx.

[25] M. L. Mangano, P. Nason and G. Ridolfi, Nucl. Phys. B 373, 295 (1992). 